Preprint typeset in JHEP style - HYPER VERSION

\title{
The Cosmophenomenology of Axionic Dark Radiation
}

\author{
Joseph P. Conlon, M.C. David Marsh \\ Rudolf Peierls Center for Theoretical Physics, 1 Keble Road \\ Oxford OX1 3NP, UK \\ Email: j.conlon1@physics.ox.ac.uk, david.marsh1@physics.ox.ac.uk
}

\begin{abstract}
Relativistic axions are good candidates for the dark radiation for which there are mounting observational hints. The primordial decays of heavy fields produce axions which are ultra-energetic compared to thermalised matter and inelastic axion-matter scattering can occur with $E_{C o M} \gg T_{\gamma}$, thus accessing many interesting processes which are otherwise kinematically forbidden in standard cosmology. Axion-photon scattering into quarks and leptons during BBN affects the light element abundances, and bounds on overproduction of ${ }^{4} \mathrm{He}$ constrain a combination of the axion decay constant and the reheating temperature. For supersymmetric models, axion scattering into visible sector superpartners can give direct non-thermal production of dark matter at $T_{\gamma} \ll T_{\text {freezeout }}$. Most axions - or any other dark radiation candidate from modulus decay — still linger today as a Cosmic Axion Background with $E_{\text {axion }} \sim \mathcal{O}(100) \mathrm{eV}$, and a flux of $\sim 10^{6} \mathrm{~cm}^{-2} \mathrm{~s}^{-1}$.
\end{abstract}




\section{Contents}

1. Introduction 1

2. Generalities of Moduli Decay

3. Axion-Photon Scattering Constraints from BBN 6

3.1 Calculation 6

3.2 Bounds on highly relativistic axions from Big Bang nucleosynthesis 8

3.2.1 Inelastic axion scattering and decaying neutral particles 8

3.2.2 Observational constraints from BBN 11

4. Dark Matter Axiogenesis 13

4.1 Scattering via Photons 14

4.2 Scattering via Gluons 16

4.3 Dark Matter Abundances 17

5. Present Day Cosmic Axion Background Flux 17

6. Conclusions 22

\section{Introduction}

Conventional cosmology begins with the Hot Big Bang. During this period, the energy density of the universe lies entirely in thermalised Standard Model degrees of freedom, and the only particles with energies $E \gg T$ are non-relativistic.

However there are mounting hints that this standard picture of the early universe is not fully correct. In particular, there may exist dark radiation, i.e. non-Standard Model particles that are relativistic at the times of Big Bang Nucleosynthesis (BBN) and at the formation of the Cosmic Microwave Background (CMB). Including the HST measurement of the Hubble constant [1], the latest observational values from WMAP, ACT, SPT and Planck are $N_{\text {eff }}=3.84 \pm 0.40$ (WMAP9, [2]), 3.71 \pm 0.35 (SPT, [3]), 3.50 \pm 0.42 (ACT, [4]), and $3.62 \pm 0.25$ (Planck, [5]). ${ }^{1}$ While by no means conclusive, these hint at a non-Standard Model value of $N_{\text {eff }}>3.046$, and the consequent existence of dark radiation.

\footnotetext{
${ }^{1}$ Without including direct measurements of $H_{0}$, the determinations using only CMB and BAO data are $N_{\text {eff }}=3.55 \pm 0.60$ (WMAP9), $2.87 \pm 0.60$ (ACT), $3.50 \pm 0.47$ (SPT) and $3.30 \pm 0.27$ (Planck). Note that there is some overlap in the data sets in all cases and so the values should not be taken as independent. Also note that the straight $\Lambda$ CDM fit to Planck gives an inferred value for $H_{0}$ of $(67.3 \pm 1.2) \mathrm{km} \mathrm{s}^{-1} \mathrm{Mpc}^{-1}$ which is low by $2 \div 3 \sigma$ compared to recent direct measurements $[1,6,7]$.
} 
Dark radiation must involve a particle that is still relativistic at CMB formation at $t \sim 3 \times 10^{5}$ years, which suggests that it is extremely light. One outstanding example of naturally light particles are axions - either the QCD axion or more generally an axionlike particle. Relativistic axions can also easily be produced non-thermally through the primordial decays of heavy moduli or saxions.

There is a large literature on the cosmology of axions and a recent general review is [8]. There is also a growing literature on dark radiation, for example [9-31]. Within these, [24] gives a general review of dark radiation from particle decay, and string models of axionic dark radiation directly relevant to this work are [22,23] (also see [19]). The role of relativistic thermal axions in dark radiation has been considered in [10,21,27].

However what this literature all tends to assume is that, once established as a dark radiation candidate, axions become non-dynamical. The justification for this is that axion scattering is suppressed by $f_{a}^{-1}$, giving $\Gamma / H \ll 1$ and an axion population which is far from thermal equilibrium. In particular, the effects of their scattering off the ambient thermal plasma are neglected.

In this paper we show that there is much interesting physics from the interactions of dark radiation axions with the thermal plasma. In particular, it allows for a violation of some familiar properties of early universe cosmology. For example, in Standard Cosmology, there are no relativistic particles with energies $E \gg T$ since the energy spectrum of particles is set by the Boltzmann distribution. The ultimate abundance of a species, $Y=n / s$, is determined either via the freeze out of stable states (as in WIMP dark matter), or by a conserved quantum number (as in the case of baryon number, where $Y_{B}$ is far in excess of the value given by thermal decoupling). Furthermore, any processes with a center of mass energy $E \gg T$ are inaccessible: the thermal bath is simply incapable of generating collisions with such a high centre of mass energy and they are suppressed by $e^{-E / T} \lll 1$.

With axionic dark radiation, these properties no longer need hold. Highly energetic axions may arise from very simple scenarios of the early universe in which long-lived heavy particles of mass $m_{\Phi}$ reheat the Standard Model degrees of freedom to $T_{\text {reheat }} \ll m_{\Phi}$, while also decaying to dark radiation axions with $E_{\text {axion }}=m_{\Phi} / 2$. The subsequent interaction of these axions with the thermalised plasma allows processes at $E_{C o M} \sim \sqrt{T_{\text {reheat }} m_{\Phi}} \gg$ $T_{\text {reheat }}$.

This scenario is very well motivated: the existence of at least one axion (the QCD axion) has strong support from the non-observation of a neutron electric dipole moment, and string theory models in general can have many 'axion-like particles' (hence, axions) which may not couple to QCD but still enjoy an approximate Peccei-Quinn shift symmetry. This symmetry forbids perturbative mass terms for the axion, making it naturally light. Furthermore axions are very weakly coupled to the visible sector - for the QCD axion, experimental bounds require $10^{9} \lesssim f_{P Q} / \mathrm{GeV}$ - and require large reheating temperatures to thermalise. For example, with $f_{P Q}=10^{10} \mathrm{GeV}$, the QCD axion thermalises at $T \gtrsim$ $2 \times 10^{7} \mathrm{GeV}$ [21]. 
The existence of a long-lived massive particle species $\Phi$ which reheats the universe through its decay is similarly well-motivated. The relative scaling of matter $\left(\rho \sim a^{-3}\right)$ and radiation $\left(\rho \sim a^{-4}\right)$ implies that, after inflation, the energy density of the universe tends to become dominated by non-relativistic matter particles with the longest lifetimes. As lifetime is inversely related to interaction strength, these are naturally particles with interactions suppressed by $M_{P l}^{-1}$ - in the context of string compactifications these are the ubiquitous moduli. Particles with Planck-suppressed couplings have a characteristic decay rate and reheat temperature

$$
\Gamma \sim \frac{1}{8 \pi} \frac{m_{\Phi}^{3}}{M_{P l}^{2}}, \quad T_{S M, \text { reheat }} \sim \frac{m_{\Phi}^{3 / 2}}{M_{P l}^{\frac{1}{2}}}=0.6 \mathrm{GeV}\left(\frac{m_{\Phi}}{10^{6} \mathrm{GeV}}\right)^{3 / 2}
$$

and will come to dominate the energy density of the universe and drive reheating. The existence of such particles is generic in string theory or any extra dimensional theory, where they arise from the higher-dimensional modes of the graviton.

However, gravity induces very general couplings and there is no a priori reason for $\Phi$ to couple only to the Standard Model. On the contrary, in general the field $\Phi$ may also decay into any light axions that exist, and such axions contribute to the 'dark radiation' of the universe. From the decay $\Phi \rightarrow a a$, the initial energy of such axions is

$$
E_{\text {axion }}=\frac{m_{\Phi}}{2} \sim T_{\text {reheat }}\left(\frac{M_{P l}}{m_{\Phi}}\right)^{\frac{1}{2}} \gg T_{\text {reheat }} .
$$

Explicit models for such decays are discussed in $[9,16,22,23]$. As axions are very weakly coupled, they do not thermalise after their original production, and their original energy is only diluted through the redshift due to the expansion of the universe. In particular, the axions remain highly energetic compared to the ambient photon plasma, by a factor $\left(M_{P l} / m_{\Phi}\right)^{\frac{1}{2}}$.

Although we have considered the particular case of Planck-coupled moduli above, the same picture will occur whenever reheating is driven by the decays of long-lived massive states (provided they have a decay mode to axions). Other examples could be topological field configurations, string winding states, Q-balls etc. The key feature is simply that $m_{\Phi} \gg T_{\text {reheat }} \sim\left(H^{2} M_{P l}^{2}\right)^{1 / 4} \sim\left(\Gamma^{2} M_{P l}^{2}\right)^{1 / 4}$. In this case the energy of hidden axions produced via direct 2-body decays of $\Phi$ is again very much greater than the particle energies in the Standard Model thermal plasma.

We also note here, that while we focus on axions in this paper, many of the considerations also apply to other dark radiation candidates such as hidden photons. The important point is only that the particles are produced from modulus decay, giving them a highly energetic spectrum compared to that of the thermalised plasma.

The fact that the great majority of axions never interact with the ambient photon plasma does not mean no interactions occur. Although such interactions may be rare, they are important because the centre of mass energy of the interaction is far higher than can be achieved by purely thermal processes. Axion-photon scattering is then the only 
way for some interactions to occur, as they are kinematically inaccessible within standard cosmology.

In this paper we study the effects of the scattering of highly energetic dark radiation axions off the ambient Standard Model plasma. We focus our discussion on two processes:

1. Inelastic scattering of axions off thermal photons into Standard Model degrees of freedom: $a+\gamma \rightarrow q_{i} \bar{q}_{i}$, where $q_{i}$ can be either a quark or a lepton. These processes can be important during and after Big Bang nucleosynthesis, and may be constrained by their effects on the light element abundances.

2. The non-thermal production of dark matter, after the conventional freeze-out temperature, by the scattering of axions off the thermal plasma and into sparticles, $a+\gamma / g \rightarrow \tilde{q} \tilde{q}^{*}$.

The paper is structured as follows. Section 2 reviews the general formalism of moduli decay to give both reheating and dark radiation. Section 3 discusses axion scattering and how it can give rise to energy injections during BBN. Section 1 describes the generation of dark matter from scattering to supersymmetric particles, and in section 5, we estimate the present day relic flux of relativistic axions. The conclusions summarise and describe possible extensions of this work.

\section{Generalities of Moduli Decay}

Throughout this paper, we assume that post-inflationary reheating is driven by the decay of a heavy modulus of mass $m_{\Phi}$ and decay rate $\Gamma$. For a Planck-coupled modulus, $\Gamma$ and $m_{\Phi}$ are related by

$$
\Gamma=\frac{1}{4 \pi} \frac{m_{\Phi}^{3}}{\left(M_{P l} / \kappa\right)^{2}},
$$

where $\kappa$ is an $\mathcal{O}(1)$ constant and $M_{P l}$ denotes the reduced Planck mass. The modulus lifetime is then

$$
\tau=\frac{4 \pi}{\kappa^{2}} \frac{M_{P l}^{2}}{m_{\Phi}^{3}} .
$$

Such a modulus is long-lived and we assume that prior to its decay it dominates the energy density of the universe. In the bulk of this paper we will use the simultaneous decay approximation in which all moduli are assumed to decay precisely at time $\tau$, although for determining the present-day axion energy spectrum in section 5 we will be more precise.

Prior to decay, the universe is matter dominated and $a \sim t^{2 / 3}$. As $H_{\text {decay }}=\frac{2}{3 t}$ during matter domination, we have

$$
H_{\text {decay }}=\frac{\kappa^{2}}{6 \pi} \frac{m_{\Phi}^{3}}{M_{P l}^{2}}
$$

We assume the modulus decays with a fraction $B_{a}$ to an axion (constituting dark radiation) and a fraction $1-B_{a}$ to the visible Standard Model sector. We will assume that the dark 
radiation component is single species and thus only consists of this axion. The initial axion energy density is then

$$
\rho_{0, \text { axion }}=B_{a} \times 3 H_{\text {decay }}^{2} M_{P l}^{2},
$$

and the initial Standard Model energy density is given by,

$$
\rho_{0, S M}=\left(1-B_{a}\right) \times 3 H_{\text {decay }}^{2} M_{P l}^{2} .
$$

As $\rho_{S M}=\frac{\pi^{2}}{30} g_{*}(T) T^{4}$, with $g_{*}(T)$ the effective number of species, we have

$$
T_{\text {reheat }}=\left(\frac{90}{\pi^{2} g_{*}\left(T_{\text {reheat }}\right)}\left(1-B_{a}\right) H_{\text {decay }}^{2} M_{P l}^{2}\right)^{1 / 4}=\kappa\left(\frac{5\left(1-B_{a}\right)}{2 \pi^{4} g_{\star}\left(T_{\text {reheat }}\right)}\right)^{1 / 4} \frac{m_{\Phi}^{3 / 2}}{M_{P l}^{1 / 2}} .
$$

The initial axion number density at time of reheating is

$$
n_{\text {axion }, 0}=B_{a} \times \frac{3 H_{\text {decay }}^{2} M_{P l}^{2}}{E_{a}}=\frac{B_{a} \kappa^{4}}{6 \pi^{2}} \frac{m_{\Phi}^{5}}{M_{P l}^{2}} .
$$

In the non-interacting limit, the energy density of the relativistic axions evolves with the size of the universe as

$$
\rho_{\text {axion }}(R)=\rho_{0, \text { axion }}\left(\frac{R_{0}}{R}\right)^{4}
$$

whereas the energy density of the Standard Model plasma evolves as

$$
\rho_{S M}(R)=\rho_{0, S M}\left(\frac{g_{*, 0}}{g_{*}}\right)^{1 / 3}\left(\frac{R_{0}}{R}\right)^{4},
$$

such that the comoving entropy $S \simeq g_{*} T^{3} R^{3}$ is conserved during the expansion of the universe. As a result the fraction of energy density in Standard Model degrees of freedom gradually increases as the universe expands and $g_{*}$ decreases. The hidden sector branching ratio $B_{a}$ relates to the experimental observable $\Delta N_{\text {eff }} \equiv N_{\text {eff }}-3.046$ as (e.g. see $[22,32]$ ),

$$
\Delta N_{\text {eff }}=\frac{43}{7} \frac{B_{a}}{1-B_{a}}\left(\frac{g_{*}\left(T_{\nu \text { decoupling }}\right)}{g_{*}\left(T_{\text {reheat }}\right)}\right)^{1 / 3} .
$$

After modulus decay, the Standard Model sector rapidly attains thermal equilibrium and the number densities and energy distribution of particles in it are set by the Boltzmann distribution. The axion decay mode $\Phi \rightarrow a a$ however produces monoenergetic particles with an energy $E_{a}=m_{\Phi} / 2$. This energy redshifts as $R^{-1}$ but does not otherwise change. ${ }^{2}$

As a numerical example we will use throughout this paper, if $m_{\Phi}=5 \times 10^{6} \mathrm{GeV}$ and $\kappa=$ 1 , then a hidden sector branching ratio of $B_{a}=0.145$ corresponds to a reheat temperature of $T_{\text {reheat }}=1.00 \mathrm{GeV}$. This branching ratio is chosen to correspond to the Planck + HST central value of $N_{\text {eff }}=3.62$, using $g_{*}\left(T_{\text {reheat }}\right)=61.75$ and $g_{*}\left(T_{\nu \text { decoupling }}\right)=10.75$. The choice of a decaying modulus mass in the region $m_{\Phi}=10^{6} \div 10^{7} \mathrm{GeV}$ is motivated by the appearance of this scale in various string scenarios [33-35]. 


\section{Particle Energies}

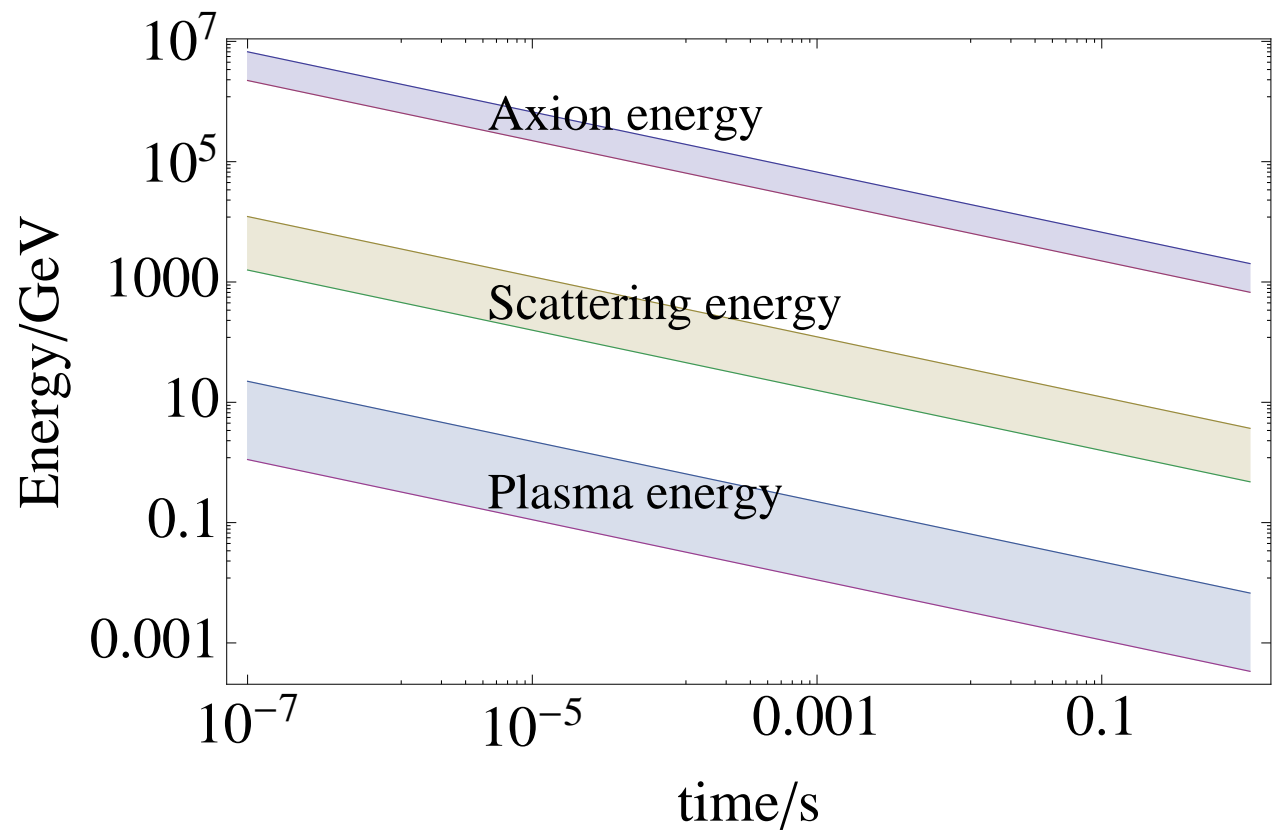

Figure 1: The three distinct energy scales and their evolution with time: the energy of the relativistic axions, the energy of the thermal Standard Model plasma, and the centre of mass energy for scattering between the axions and the thermal plasma. These can be separated by several orders of magnitude.

In this paper, our main interest will lie in the scattering of the relativistic axions off the thermal Standard Model plasma. In figure 1 we illustrate the approximate distribution of energies as the universe evolves, and how they differ from standard cosmology.

\section{Axion-Photon Scattering Constraints from BBN}

In this section we compute the inelastic scattering rate for an axion off thermal photons and determine the resulting constraints due to energy injections during BBN. We consider the process,

$$
a+\gamma \rightarrow q+\bar{q},
$$

for the fermion/antifermion pair $q \bar{q}$ as placeholder for processes including axion scattering into quarks, $a+\gamma \rightarrow q_{i}+\bar{q}_{i}$, or any leptons, $a+\gamma \rightarrow l_{i}^{-}+l_{i}^{+}$.

\subsection{Calculation}

The potential Lagrangian interaction terms are

$$
\text { Axion-fermion-fermion: } \quad c_{q} \frac{m_{q}}{f_{a}} a \bar{\psi} \gamma^{5} \psi,
$$

\footnotetext{
${ }^{2}$ The monoenergetic spectrum is a feature of the instantaneous decay approximation. In practice the decays will occur at different times and the spectrum will be smeared by redshifting.
} 

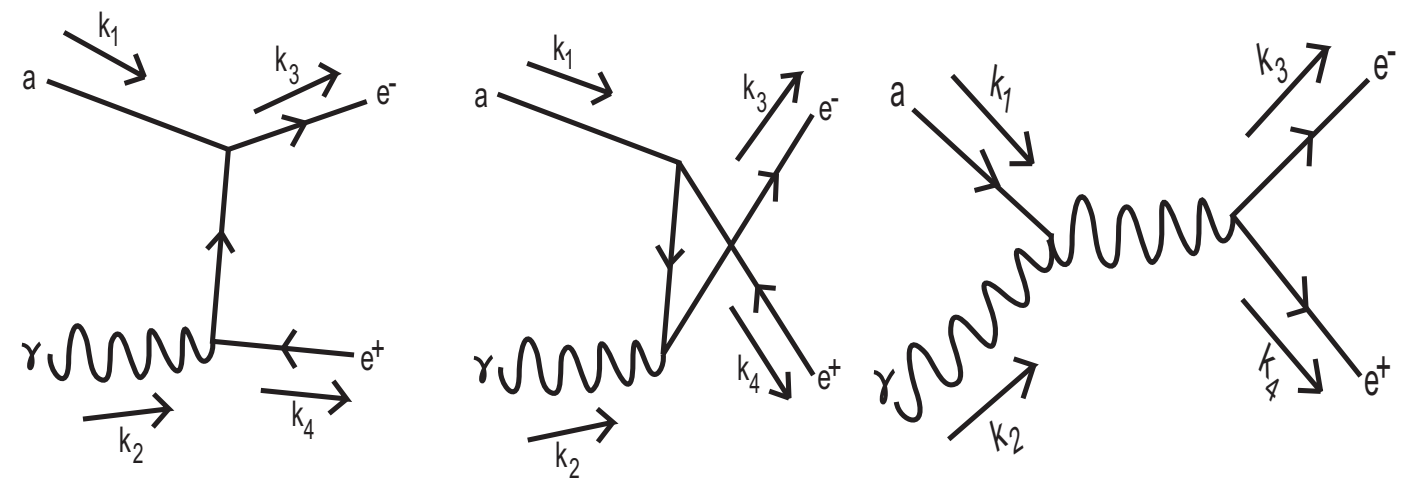

Figure 2: Feynman diagrams contributing to $a+\gamma \rightarrow q \bar{q}$. The third diagram involves an additional factor of $\alpha_{E M}$ and so we neglect it.

$$
\begin{aligned}
\text { Photon-fermion-fermion: } & q_{q} A_{\mu} \bar{\psi} \gamma^{\mu} \psi, \\
\text { Axion-photon-photon: } & c_{\gamma} \frac{\alpha_{e m}}{8 \pi} \frac{a}{f_{a}} \epsilon_{\mu \nu \lambda \rho} F^{\mu \nu} F^{\lambda \rho} \equiv g_{a \gamma \gamma} a \mathbf{E} \cdot \mathbf{B},
\end{aligned}
$$

where $c_{q}$ and $c_{\gamma}$ are model-dependent constants. We shall henceforth take $c_{q}=1$. The process (3.1) proceeds via the Feynman diagrams shown in figure 2. As the third diagram involves the $a \gamma \gamma$ vertex which is suppressed by an additional factor of $\frac{\alpha_{e m}}{4 \pi}$, we shall not consider it further here. ${ }^{3}$

We aim to compute the axion scattering rate,

$$
\Gamma_{a \gamma \rightarrow q \bar{q}}=\Gamma=\langle n \sigma v\rangle,
$$

by first computing $\sigma v$, averaged over final state momenta and initial directions, for an axion of energy $E_{a}$ scattering on a photon of energy $E_{\gamma}$, and then subsequently performing the thermal averaging over $E_{\gamma}$.

The axion and photon 4-momenta are

$$
\begin{aligned}
k_{\text {axion }} & =\left(E_{a}, E_{a}, 0,0\right), \\
k_{\text {photon }} & =\left(E_{\gamma}, E_{\gamma} \cos \theta, E_{\gamma} \sin \theta, 0\right) .
\end{aligned}
$$

where we have assumed $m_{a} \ll E_{a}$. The centre of mass energy is then given by $E_{C o M}^{2}=$ $2 k_{\text {axion }} \cdot k_{\text {photon }}=2 E_{a} E_{\gamma}(1-x)$ with $x=\cos \theta$.

Denoting the amplitudes for the first two diagrams in figure 2 by $\mathcal{M}_{A}$ and $\mathcal{M}_{B}$ respectively, we have

$$
\frac{1}{2} \sum_{\text {spins }}\left|\mathcal{M}_{A}+\mathcal{M}_{B}\right|^{2}=\frac{2 q_{q}^{2} m_{q}^{2}}{f_{a}^{2}}\left(\frac{u}{t}+\frac{t}{u}+2\right),
$$

where the first term arises from $\left|\mathcal{M}_{A}\right|^{2}$, the second from $\left|\mathcal{M}_{B}\right|^{2}$, and the third from the cross term. Here $t=k_{1} \cdot k_{3}$ and $u=k_{1} \cdot k_{4}$. Integrating over the $q \bar{q}$ phase space, we get

$$
\sigma=\frac{1}{4 E_{a} E_{\gamma}\left|v_{a}-v_{\gamma}\right|} \frac{q_{q}^{2} m_{q}^{2}}{2 \pi f_{a}^{2}} \ln \left(\frac{E^{\prime}+p^{\prime}}{E^{\prime}-p^{\prime}}\right) .
$$

\footnotetext{
${ }^{3}$ The presence of $\alpha_{E M}$ can be understood by starting with the 'natural' form of the gauge kinetic action, $\frac{1}{4 g_{E M}^{2}} F_{\mu \nu} F^{\mu \nu}+\frac{a}{f_{a}} F_{\mu \nu} \tilde{F}^{\mu \nu}$, and then canonically normalising the kinetic terms.
} 
Here $E^{\prime}=E_{C o M} / 2$ is the energy of the fermion in the centre of mass frame and $p^{\prime}=$ $\sqrt{\left(E^{\prime}\right)^{2}-m_{q}^{2}}$ is the momentum of the fermion in the centre of mass frame.

We can average $\sigma v$ over the initial photon direction by performing the $x$-integral, $\frac{1}{2} \int_{-1}^{1-\lambda} d x$, where $\lambda=\frac{2 m_{q}^{2}}{E_{a} E_{\gamma}}$ accounts for the threshold energy for $q \bar{q}$ pair production. The relevant integral is

$$
\begin{aligned}
\langle\sigma v\rangle & =\frac{q_{q}^{2} \lambda}{16 \pi f_{a}^{2}} \int_{-1}^{1-\lambda} d x \ln \left(\frac{\sqrt{1-x}+\sqrt{1-x-\lambda}}{\sqrt{1-x}-\sqrt{1-x-\lambda}}\right)= \\
& \frac{q_{q}^{2} \lambda}{16 \pi f_{a}^{2}}\left(-\sqrt{4-2 \lambda}+(\lambda-2) \log (\sqrt{2}-\sqrt{2-\lambda})+2 \log (\sqrt{2-\lambda}+\sqrt{2})-\frac{1}{2} \lambda \log (\lambda)\right) .
\end{aligned}
$$

Finally, we need to integrate $\langle\sigma v\rangle$ over the photon spectrum using the thermal number density,

$$
d n=\frac{g}{2 \pi^{2}} \frac{E_{\gamma}^{2} d E_{\gamma}}{e^{\frac{E_{\gamma}}{T}}-1},
$$

with $g=2$. Using $E_{\gamma}=\frac{2 m_{q}^{2}}{E_{a} \lambda}$, we can rewrite $\frac{E_{\gamma}^{2} d E_{\gamma}}{e^{E \gamma / T}-1}=\frac{d \lambda}{e^{2 m_{q}^{2}}} \frac{8 m_{e}^{6}}{E^{3} \lambda^{4}}$, with $\lambda$ running from 0 to 2 . We then end up with,

$$
\begin{aligned}
\Gamma=\langle n \sigma v\rangle & =\frac{g}{2 \pi^{2}} \frac{m_{q}^{6}}{E_{a}^{3}} \frac{q_{q}^{2}}{2 \pi f_{a}^{2}} \int_{0}^{2} d \lambda \frac{1}{e^{\frac{2 m_{q}^{2}}{E_{a} \lambda T}}-1} \frac{1}{\lambda^{3}}(-\sqrt{4-2 \lambda}+ \\
& \left.+2 \log (\sqrt{2-\lambda}+\sqrt{2})-(2-\lambda) \log (\sqrt{2}-\sqrt{2-\lambda})-\frac{1}{2} \lambda \log (\lambda)\right),
\end{aligned}
$$

which can be evaluated numerically as a function of the relevant parameters.

\subsection{Bounds on highly relativistic axions from Big Bang nucleosynthesis}

Equation (3.7) gives the scattering rate of energetic axions off thermal photons, and in this section we will show how this scattering rate - despite its relative smallness in relation to the Hubble parameter in the early universe - can be large enough to affect the primordial ${ }^{4}$ He abundance.

After the decay of the modulus $\Phi$ into Standard Model degrees of freedom and axions, the number density of axions is diluted with the expansion of space-time. Occasional scattering of axions off the thermal plasma into 'primary' scattering products such as $q \bar{q}$ or quark/anti-quark pairs, quickly thermalize by producing a large number of electromagnetic (i.e. $\gamma, e^{ \pm}$) or hadronic (e.g. $\left.p, n\right)$ 'secondary' particles. The secondaries may in turn affect the light element abundances in the early universe through a number of different processes, as described in detail in e.g. [36-39]. This way, relativistic axions may in principle be constrained by Big Bang Nucleosynthesis bounds on nuclear abundances.

\subsubsection{Inelastic axion scattering and decaying neutral particles}

Unfortunately, to our knowledge there exists no dedicated study of what constraints may be inferred from light element yields during BBN on a highly relativistic species which 
scatters inelastically off the thermal plasma. However, there are well-known constraints on massive particle species, here denoted $X$, decaying with life-time $\tau_{X}$ during and after BBN. These bounds are often quoted in terms of

$$
\epsilon_{X} \equiv M_{X} \xi_{X}
$$

where $\xi_{X}=n_{X} / n_{\gamma}$ is the relative abundance of $X$-particles to photons prior to the decay, and $M_{X}$ is the mass of $X$. The quantity $\epsilon_{X}$ measures the total energy deposited per photon once the entire species $X$ has decayed. Axion scattering off the thermal plasma differs from a decaying particle in four ways:

1. The scattering rate, and thereby the effective 'life-time' $\left(\tau=\Gamma^{-1}\right)$, depends on the temperature of the thermal photons as well as the energy of the axion, which both decrease with time. This gives rise to a particular time-dependence for the scattering rate into each primary final state.

2. While the decay products of a decaying massive particle are traditionally assumed to have negligible total momentum with respect to the rest frame of the plasma, the primaries of axion scattering will necessarily appear boosted with respect to this frame. Yet, the average total momentum over many inelastic axion scatterings will vanish, and we expect this point to be of less importance to the applicability of the bounds.

3. The role of the factor of $M_{X}$ in $\epsilon_{X}$ which determines the total energy of the decay products is in the case of axion scattering played by the time-dependent axion energy $E_{a} \gg T$.

4. Finally, while the number of $X$-particles is greatly reduced at times $t / \tau_{X} \gg 1$, the number density of axions per comoving volume does not change by much for the scattering rates that we will discuss. In other words, axion scatterings are relatively rare events, and most relativistic axions pass through BBN unperturbed.

Despite these obvious differences, we find that at the level of the energy deposition history - which is in the end what is constrained by the BBN analysis - each axion scattering channel can be well approximated by a species of decaying particle. Thus, the effects of the axion during BBN can be modelled by a collection of decaying particle species, each decaying only to a particular final state $q_{i} \bar{q}_{i}$ with life-time $\tau_{i}$ and deposited energy (per photon) $\epsilon_{i}$. The corresponding hadronic branching ratio for each of these scattering modes will be either 1 (for quark and heavy lepton primaries) or $\sim 10^{-3}$ (for electron primaries).

In this section, we impose known bounds on the energy deposition of each individual such species during $\mathrm{BBN}$, and interpret the bound as a constraint on the axion decay constant and modulus mass $m_{\Phi}$. These bounds are necessarily conservative as the effect of each possible primary is considered separately, yet we will see how they can give important constraints on the axion decay constant. 
Specifically, constraints on a neutral decaying particle during (and after) BBN are conveniently phrased in terms of bounds on

$$
\epsilon_{X}=-\int_{0}^{\infty} d t^{\prime} M_{X} \dot{\xi}_{X}\left(t^{\prime}\right)=\int_{0}^{\infty} d t^{\prime} M_{X}\left|\Gamma_{X}\right| \xi_{X}\left(t^{\prime}\right)
$$

given the life-time $\tau_{X}$. For axion scattering into a particular final state, here denoted $q \bar{q}$, the corresponding bounds can be phrased in terms of

$$
\epsilon_{a}=\int_{t_{0}}^{\infty} d t^{\prime} E_{a}\left(t^{\prime}\right)\left|\Gamma_{a \gamma \rightarrow q \bar{q}}\left(t^{\prime}\right)\right| \xi_{a}\left(t^{\prime}\right)
$$

where $\xi_{a}=n_{a} / n_{\gamma}$ denotes the relative number density of axions to photons. Here $t_{0}$ denotes an initial time before which no bounds can be placed on the energy deposition, and during radiation domination, $E_{a}\left(t^{\prime}\right)=E_{0}\left(\frac{t_{r h}}{t^{\prime}}\right)^{1 / 2}$. Thus, the deposited energy from axion scattering into $q \bar{q}$-pairs from the time $t_{0}$ up to $t$ is given by,

$$
\epsilon_{a}(t)=E_{0} \int_{t_{0}}^{t} d t^{\prime}\left(\frac{t_{r h}}{t^{\prime}}\right)^{1 / 2}\left|\Gamma_{a \gamma \rightarrow q \bar{q}}\left(t^{\prime}\right)\right| \xi_{a}\left(t^{\prime}\right) .
$$

Over a period in which the effective number of species remains the same, the temperature of the photon gas depends on time as $T=T_{r h} \sqrt{t_{r h} / t}$, and the re-heating temperature is determined from the mass of the decaying modulus (and thereby $E_{0}$ ) as in equation (2.6).

Thus, the energy deposition for axion scattering exclusively into $q \bar{q}$ can be found explicitly, and an example thereof is shown in figure 3 for scattering into bottom quarks. From this figure, we note that the energy deposition history from axion scattering is very similar to that of a decaying particle species, $\epsilon_{X}(t)=M_{X}\left(\xi_{X}\left(t_{0}\right)-\xi_{X}(t)\right)$. This allows us to map each axion scattering mode into a species of neutral decaying particles with life-time, mass and initial number density determined by optimising the fit of the energy deposition profile to that of the corresponding axion scattering.

However, before turning to the detailed constraints obtained by carefully evaluating the analysis presented in this section, we will now give an order of magnitude estimate of the energy deposition $\epsilon_{a}$ for the particular case in which $m_{\Phi}=2 \cdot 10^{6} \mathrm{GeV}$, and the axions give rise to an excess radiation of $\Delta N_{\text {eff }}=0.57$. In this case, the effective 'life-time' for axion-photon scattering into a $b \bar{b}$ pair is $\tau \approx 0.2 \mathrm{~s}$, at which time the temperature of the plasma is $T \approx 4 \mathrm{MeV}$ and $E_{a} \approx 16 \mathrm{TeV}$. For this rough estimate, we evaluate the scattering cross-section by taking $f_{a}=10^{9} \mathrm{GeV}$ and $E_{\gamma}=T$ in equation (3.5), and only consider axion-photon scattering at a $90^{\circ}$ angle. Upon substituting the fermion mass in equation (3.5) by the bottom quark mass, we find that

$$
\sigma_{\text {approx }} \approx 6 \cdot 10^{-28} \mathrm{MeV}^{-2} .
$$

For this order of magnitude estimate, we note that $\rho_{a}=\Delta N_{\text {eff }} \rho_{\nu}=\Delta N_{\text {eff }} \frac{7}{8} \frac{\pi^{2}}{15} T^{4}=$ $0.58(4 \mathrm{MeV})^{4}$, where $\rho_{\nu}$ denotes the energy density of a single neutrino species at $T=4$ $\mathrm{MeV}$. Similarly, we approximate the Hubble parameter to be $H \approx 7.2 \cdot 10^{-21} \mathrm{MeV}$ at this 


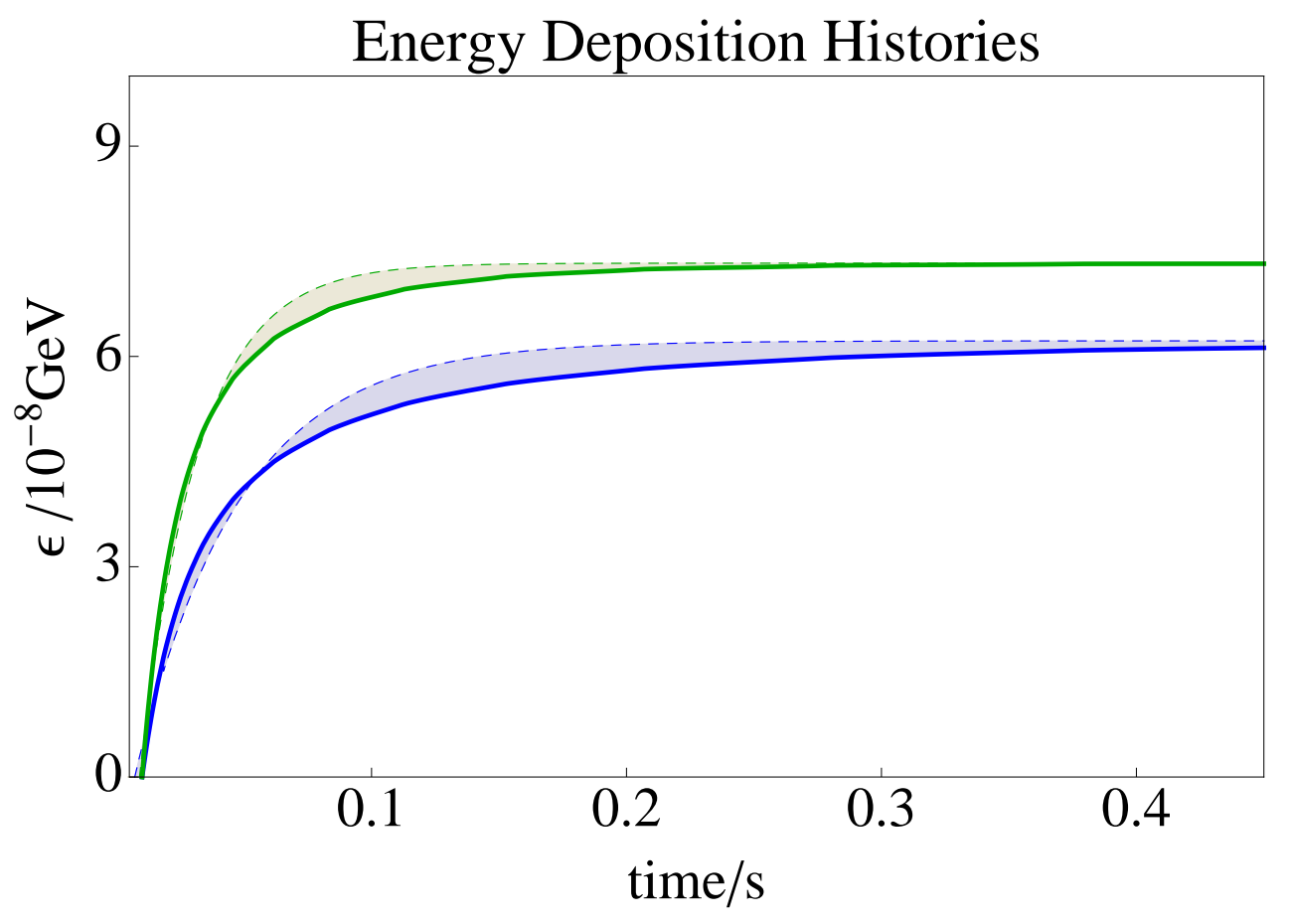

Figure 3: Energy deposition histories $\epsilon_{a}(t)$ for axion inelastic scattering to bottom quarks (solid lines) as compared to the best-fit profile of $\epsilon_{X}(t)$ for a decaying particle species (dashed lines). For both solid curves, $f_{a}=10^{9} \mathrm{GeV}$ and $\Delta N_{\text {eff }}=0.57$. In blue, $m_{\Phi}=5 \cdot 10^{6} \mathrm{GeV}$, and in green $m_{\Phi}=5 \cdot 10^{7} \mathrm{GeV}$.

time. The energy deposition from axion-photon scattering into $b \bar{b}$ quarks may thus be estimated as

$$
\epsilon_{a} \approx \frac{1}{H n_{\gamma}} \sigma_{\text {approx }} \rho_{a} n_{\gamma} \approx 1 \cdot 10^{-8} \mathrm{GeV}
$$

From figure 6 of [38], we find that the bound on $\epsilon_{X}$ for a decaying neutral particle of mass $M_{X}=1 \mathrm{TeV}$ at $t=0.2 \mathrm{~s}$ is given by $\epsilon_{X}<4 \cdot 10^{-8} \mathrm{GeV}$. Thus, based on this heuristic estimate, we conclude that inelastic axion-photon scattering may deposit significant amounts of energy in the thermal plasma during BBN, and we will now proceed by a more careful evaluation of the corresponding bounds on the axion decay constant.

\subsubsection{Observational constraints from $\mathrm{BBN}$}

During BBN $\left(10^{-2} \lesssim t \lesssim 10^{2}\right.$ seconds), the strongest constrains apply to processes which increase the relative number of neutrons to protons as these neutrons will in turn increase the Helium abundance, $Y_{p}$. At the very beginning of this period, $t \lesssim 3 \cdot 10^{-2}$ seconds, even these bounds are ineffective as thermal weak interactions quickly restore the neutron to proton ratio. However, particles decaying later during BBN may be constrained from bounds on overproduction of ${ }^{4} \mathrm{He}$. 


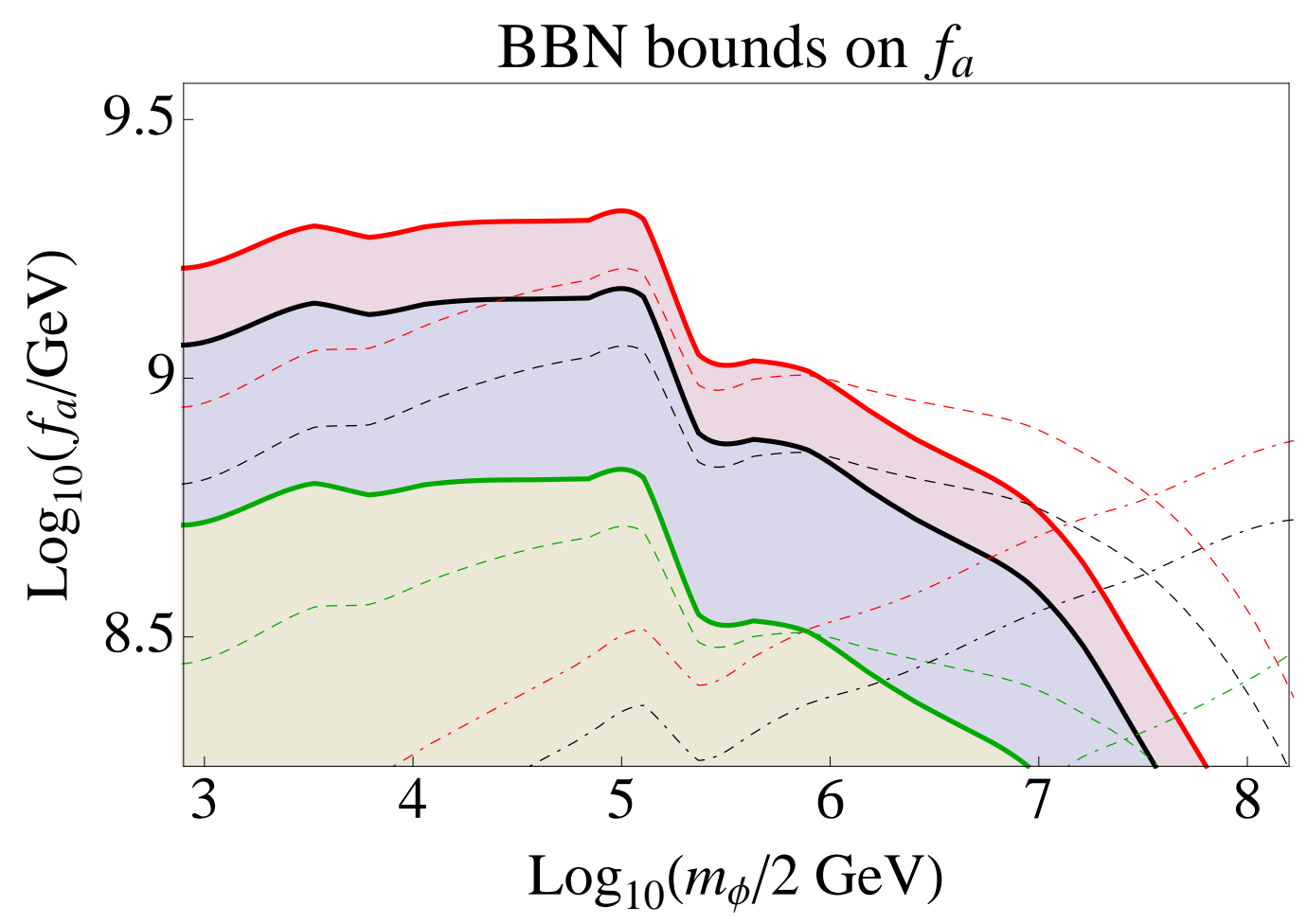

Figure 4: Conservative bounds on the axion decay constant as a function of the initial axion energy obtained by considering the effects of the decay channels to $b \bar{b}$ (solid), $c \bar{c}$ (dashed), and $s \bar{s}$ (dot-dashed) separately. The red, black and green curves correspond to $\Delta N_{\text {eff }}=0.1,0.5$ and 1 , respectively, and the areas below the curves are excluded from the constraints from [38] due to overproduction of ${ }^{4} \mathrm{He}$ during $\mathrm{BBN}$.

The primordial Helium abundance can be inferred both from spectroscopy of extra galactic $\mathrm{H}_{\mathrm{II}}$ regions by extrapolation to zero metallicity, as well as from fits of cosmological models of the temperature anisotropies of the $\mathrm{CMB}$ and the large-scale structure of the universe (for a recent review, see [40]). Recent spectroscopic studies have found $Y_{p}^{B B N}=$ $0.2565 \pm 0.0010$ (stat) \pm 0.0050 (syst) $[41], Y_{p}^{B B N}=0.2528 \pm 0.0028$ [42] , and $Y_{p}^{B B N}=$ $0.2534 \pm 0.0083$ [43]. These values are compatible within $1 \sigma$ to those inferred from the recent Planck experiment, which estimated $Y_{p}^{B B N}=0.266 \pm 0.021$ for the $\Lambda$ CDM model extended with $Y_{p}^{B B N}$ as a free parameter, and $Y_{p}^{B B N}=0.254_{-0.033}^{+0.041}$ for $\Lambda \mathrm{CDM}$ extended with both $Y_{p}^{B B N}$ and $N_{\text {eff }}$ (the latter parameter is then determined to $N_{\text {eff }}=3.33_{-0.83}^{+0.59}$ ). In this paper, we will use the constraints on decaying neutral particles from [38] which are based on the conservative bound of $Y_{p}<0.258$.

Given the resulting constraint on $\xi_{X}$ for a given life-time, a (conservative) bound on the energy deposited by the scattering axion into each decay channel may be derived. The resulting constraints as obtained separately for the $b \bar{b}, c \bar{c}$ and $s \bar{s}$ scattering channels are shown in figure 4 . We note that these constraints probe an interesting region of the axion parameter space, and that dedicated studies of inelastic axion scattering during BBN may well be able to derive even stronger bounds on $f_{a}$. 


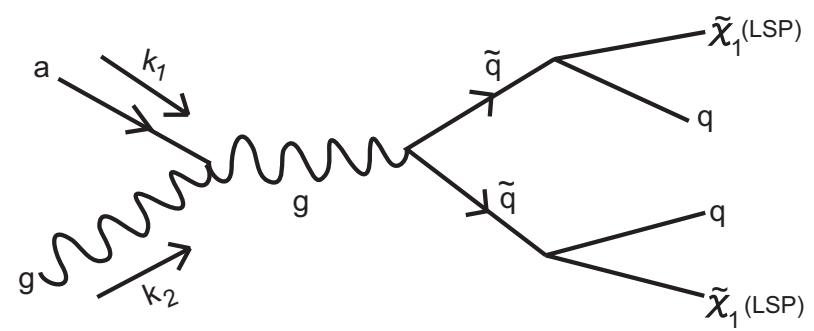

Figure 5: Dark matter production from axion scattering. Here the axion scatters off a gluon, leading to pair production of heavy squarks which then cascade decay to produce the LSP.

\section{Dark Matter Axiogenesis}

One popular model of dark matter is that it consists of the lightest supersymmetric particle (LSP) whose stability is protected by R-parity. Conventionally, the abundance of the LSP is determined via a thermal freeze out from the ambient plasma at a temperature of approximately $T_{\text {freeze-out }} \simeq m_{L S P} / 20$.

However, the scenario considered here offers a radically new production mechanism. Even when the thermal plasma has $T<T_{\text {freeze-out }} \ll m_{L S P}$, energetic axionic dark radiation can directly produce supersymmetric particles through scattering off the ambient plasma. The produced susy particles will undergo a rapid cascade decay to the LSP, which will remain as a stable dark matter relic. A sample process leading to dark matter production is shown in figure 5. The rate of dark matter production is set by the axion scattering rate. This is an entirely non-thermal process, and can give significant LSP production at temperatures $T \ll T_{\text {freeze-out }}$.

Let us first give the basic scaling estimates for the dark matter abundance produced (a proper calculation follows). For the estimate we assume the axion-plasma center of mass energy is above threshold for producing susy particles and neglect mass effects. We also use only the 'universal' $a \gamma \gamma$ or agg vertex shown in figure 5, so there is no mass suppression of the axion-matter coupling.

The density of plasma particles is $n \sim T^{3}$ and the axion scattering cross-section is $\sigma \sim\left(\frac{\alpha}{4 \pi} \frac{1}{f_{a}}\right)^{2}$. The total scattering rate of an axion with the ambient plasma is then

$$
\Gamma=\langle n \sigma v\rangle \sim\left(\frac{\alpha}{4 \pi}\right)^{2} \frac{T^{3}}{f_{a}^{2}}
$$

As during radiation domination $T \sim t^{-1 / 2}$, the scattering rate $\Gamma \sim t^{-3 / 2}$ and it is clear that the total number of scattering events $\mathcal{N}_{\text {total }}=\int \Gamma d t$ is dominated by those occurring at early times. As the Hubble scale is $H \sim \frac{T^{2}}{M_{P l}}$, the fraction of axions that interact in a Hubble time is

$$
\frac{\Gamma}{H} \sim \frac{T M_{P l}}{f_{a}^{2}}\left(\frac{\alpha}{4 \pi}\right)^{2} .
$$

As this is dominated by early times, in the instantaneous-reheating approximation, we simply evaluate this at $T \sim T_{\text {reheat }}$ to get an estimate of the total fraction of axions 
that scatter. Each decaying modulus of mass $m_{\Phi}$ generates either two axions of energies $E_{a}=m_{\Phi} / 2$ or $\sim m_{\Phi} / T_{\text {reheat }}$ thermalised Standard Model particles. If the axion branching ratio is $B_{a}$, the axion abundance is

$$
Y_{\text {axion }} \equiv \frac{n_{\text {axion }}}{s} \sim \frac{B_{a} T_{\text {reheat }}}{m_{\Phi}}
$$

As each axion scattering event to R-parity odd particles produces two dark matter particles, the resulting dark matter abundance is

$$
Y_{d m} \sim Y_{\text {axion }} \frac{2 \Gamma}{H} \sim B_{a} \frac{T_{r e h e a t}^{2} M_{P l}}{m_{\Phi} f_{a}^{2}}\left(\frac{\alpha}{4 \pi}\right)^{2}
$$

While this neglects factors of $g_{*}, \pi$ etc, it is clear that for reasonable parameter values this can be a phenomenologically interesting source of dark matter (note that $Y_{d m} \sim 1.5 \times 10^{-12}$ for a $300 \mathrm{GeV} \mathrm{LSP).}$

Overall, any source of axion-plasma interaction that produces susy particles can produce dark matter. Such diagrams are shown in figure 6. We will return to the full set of diagrams of figure 6 in future work. Here however we focus only on the universal diagrams involving s-channel photons or gluons. These are universal as the coupling agg is necessarily present for a QCD axion, whereas any interactions with quarks or squarks are more model dependent with unspecified coefficients.

\subsection{Scattering via Photons}

We consider the scattering process $a+\gamma \rightarrow \tilde{q} \tilde{q}^{*}$ via an s-channel photon. We treat the axion as massless, and give the squarks a mass of $m_{\tilde{q}}$. In section 4.2 we will consider the analogous process $a+g \rightarrow \tilde{q} \tilde{q}^{*}$. The $a \gamma \gamma$ interaction is

$$
\mathcal{L}_{a \gamma \gamma}=a g_{a \gamma \gamma} \mathbf{E} \cdot \mathbf{B} \equiv \frac{1}{8} a g_{a \gamma \gamma} \epsilon_{\mu \nu \lambda \rho} F^{\mu \nu} F^{\lambda \rho} .
$$

The resulting amplitude is,

$$
\mathcal{M}=e g_{a \gamma \gamma} \epsilon_{\mu \nu \lambda \rho} \frac{k_{2}^{\mu} k_{1}^{\lambda} \epsilon^{\nu}\left(k_{3}-k_{4}\right)^{\rho}}{\left(k_{1}+k_{2}\right)^{2}}
$$

where $k_{1}$ and $k_{2}$ are the incident 4-momenta of the axion and photon, and $k_{3}$ and $k_{4}$ the 4 -momenta of the outgoing squarks or sleptons. In the center of mass frame we can without loss of generality write

$$
\begin{aligned}
k_{1} & =\left(E_{C M}, 0,0, E_{C M}\right), \\
k_{2} & =\left(E_{C M}, 0,0,-E_{C M}\right), \\
k_{3} & =\left(E_{C M}, 0, p \sin \theta, p \cos \theta\right), \\
k_{4} & =\left(E_{C M}, 0,-p \sin \theta,-p \cos \theta\right), \\
\epsilon & =(0, \cos \phi, \sin \phi, 0),
\end{aligned}
$$




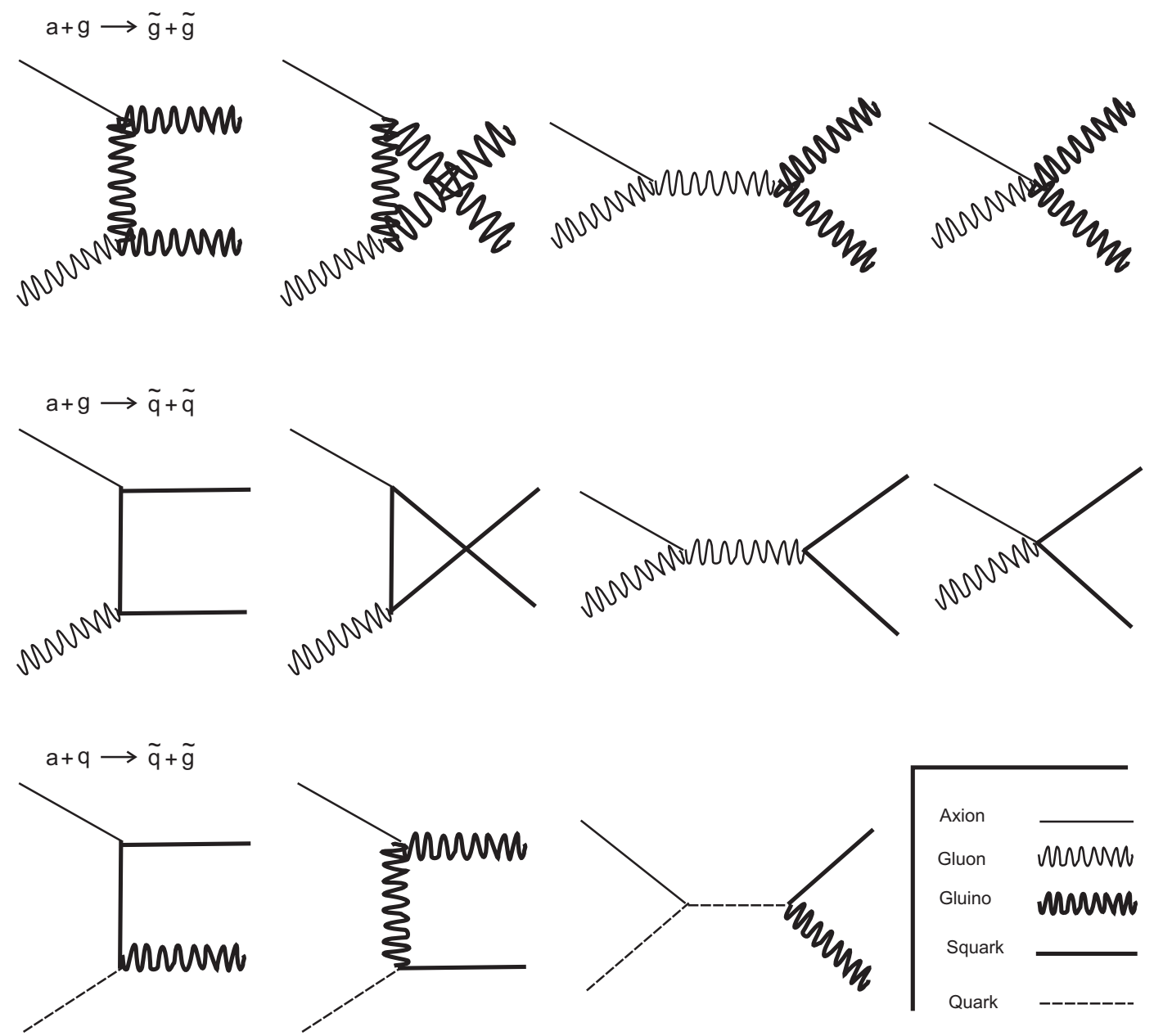

Figure 6: Feynman diagrams that lead to LSP production from axion scattering off the ambient plasma. We have shown diagrams that involve strong interactions but there are also similar diagrams involving weak or electromagnetic scattering off photons or leptons.

giving

$$
|\mathcal{M}|^{2}=e^{2} g_{a \gamma \gamma}^{2} p^{2} \cos ^{2} \phi \sin ^{2} \theta .
$$

Averaging over the initial photon polarisation then gives

$$
|\mathcal{M}|^{2}=\frac{e^{2} g_{a \gamma \gamma}^{2}}{2} p^{2} \sin ^{2} \theta
$$

If the lab frame 4-momenta are

$$
\begin{aligned}
& k_{a}=(E, 0,0, E), \\
& k_{\gamma}=\left(E_{\gamma}, 0, E_{\gamma} \sin \theta, E_{\gamma} \cos \theta\right),
\end{aligned}
$$

then the resulting cross-section, integrated over final state phase space, is

$$
\sigma v=\frac{1}{4 E E_{\gamma}} \frac{e^{2} g_{a \gamma \gamma}^{2}}{24 \pi} \sqrt{1-\frac{2 m^{2}}{E E_{\gamma}(1-x)}}\left(\frac{E E_{\gamma}}{2}(1-x)-m^{2}\right),
$$


where $x=\cos \theta$. We finally average over the relative angle between the photon and axion, $\langle\sigma v\rangle=\frac{1}{2} \int_{-1}^{1-\lambda} \sigma v d x$. In terms of $\lambda=\frac{2 m^{2}}{E E_{\gamma}}$, this is

$$
\langle\sigma v\rangle=\frac{1}{128} \frac{e^{2} g_{a \gamma \gamma}^{2}}{24 \pi}\left(2(4-5 \lambda) \sqrt{4-2 \lambda}+6 \lambda^{2} \ln (\sqrt{2}+\sqrt{2-\lambda})-3 \lambda^{2} \ln \lambda\right),
$$

for the x-section $\langle\sigma v\rangle$ of an axion of energy $E$ incident on a single photon of energy $E_{\gamma}$. We finally sum over the spectrum of thermal photons by integrating

$$
\frac{g^{2}}{2 \pi^{2}} \int \frac{E_{\gamma}^{2}}{e^{E_{\gamma} / T}-1} d E_{\gamma}=\frac{g^{2}}{2 \pi^{2}} \int_{0}^{2} d \lambda \frac{1}{e^{\frac{2 m^{2}}{E \lambda T}}-1} \frac{8 m^{6}}{E^{3} \lambda^{4}} .
$$

The scattering rate to a pair of charged scalars is finally

$$
\begin{aligned}
\Gamma=\langle n \sigma v\rangle= & \frac{g^{2}}{2 \pi^{2}} \int_{0}^{2} d \lambda \frac{1}{e^{\frac{2 m^{2}}{E \lambda T}}-1} \frac{8 m^{6}}{E^{3} \lambda^{4}} \times \frac{1}{128} \frac{e^{2} g_{a \gamma \gamma}^{2}}{24 \pi}(2(4-5 \lambda) \sqrt{4-2 \lambda} \\
& \left.+6 \lambda^{2} \ln (\sqrt{2}+\sqrt{2-\lambda})-3 \lambda^{2} \ln \lambda\right) .
\end{aligned}
$$

We obtain the total scattering rate to sleptons and squarks by summing over all species. For simplicity, we assume a common mass scale for all squarks and sleptons. Writing $e=q \sqrt{\frac{4 \pi}{137}}$, we have

$$
\Gamma_{\text {total }}=\underbrace{6 \times \Gamma(q=1)}_{\text {sleptons }}+\underbrace{6 \times 3 \times \Gamma(q=2 / 3)}_{\text {up squarks }}+\underbrace{6 \times 3 \times \Gamma(q=1 / 3)}_{\text {down squarks }} .
$$

As each axion scattering event produces pairs of susy particles, each of which undergoes a rapid cascade decay down to the LSP, the overall LSP production rate is

$$
\Gamma_{L S P}=2 \times \Gamma_{\text {total }} .
$$

\subsection{Scattering via Gluons}

There is also a kinematically identical diagram where the s-channel photon is replaced by a gluon. This differs only by the colour factors and the conventional notation for axionphoton or axion-gluon couplings. The agg vertex comes from the interaction

$$
\frac{a}{f_{P Q}} \frac{\alpha_{s}}{16 \pi} \epsilon_{\alpha \beta \gamma \delta} G_{a}^{\alpha \beta} G_{a}^{\gamma \delta} \in \mathcal{L} .
$$

By comparison of eq. (4.11) with eq. (4.2), we see that we have to replace

$$
\left(g_{a \gamma \gamma}\right)_{\text {photon }} \text { with }\left(\frac{1}{f_{P Q}} \frac{\alpha_{s}}{2 \pi}\right)_{\text {strong }}
$$

and

$$
-i e\left(k_{3}-k_{4}\right)^{\mu} \text { with }-i g_{s}\left(k_{3}-k_{4}\right)^{\mu} t^{b},
$$

where $g_{s}$ is the strong coupling and $\alpha_{s}=\frac{g_{s}^{2}}{4 \pi}$. Summing over the final squark colour states, in eq. (5.11) we replace

$$
e^{2} g_{a \gamma \gamma}^{2} \text { with }\left(\frac{1}{f_{P Q}} \frac{\alpha_{s}}{2 \pi}\right)^{2} \frac{g_{s}^{2}}{2} .
$$

As all possible scalar final states are squarks, we have

$$
\Gamma_{\text {total }}=12 \times \Gamma \text {, and again } \Gamma_{L S P}=2 \times \Gamma_{\text {total }} .
$$




\subsection{Dark Matter Abundances}

In the instantaneous reheating approximation, we will take $\frac{\Gamma\left(T_{\text {reheat }}\right)}{H\left(T_{\text {reheat }}\right)}$ as a proxy for the total fraction of axions that scatter. The resulting dark matter number density is

$$
n_{L S P}=2 \times n_{\text {axion }}\left(T_{\text {reheat }}\right) \times \frac{\Gamma\left(T_{\text {reheat }}\right)}{H\left(T_{\text {reheat }}\right)} .
$$

The axion number density is given by eq. (2.7) which we repeat here,

$$
n_{\text {axion }, 0}=B_{a} \times \frac{3 H\left(T_{\text {reheat }}\right)^{2} M_{P l}^{2}}{E_{a}},
$$

with $B_{a}$ the modulus branching ratio to axions.

As the entropy density is $s=\frac{2 \pi^{2}}{45} g_{*} T^{3}$, the dark matter abundance is given by

$$
Y_{L S P} \equiv \frac{n_{L S P}}{s}=\frac{45}{g_{*} \pi^{2}} \times \frac{n_{\text {axion }}\left(T_{\text {reheat }}\right)}{T_{\text {reheat }}^{3}} \times \frac{\Gamma\left(T_{\text {reheat }}\right)}{H\left(T_{\text {reheat }}\right)} .
$$

$\Gamma\left(T_{\text {reheat }}\right)$ is set by eq. (4.12). $H\left(T_{\text {reheat }}\right)$ is determined by taking

$$
\Gamma_{\Phi \rightarrow \text { all }}=\frac{1}{4 \pi} \frac{m_{\Phi}^{3}}{\left(M_{P l} / \kappa\right)^{2}},
$$

where $\kappa$ is a parameter we scan over.

We scan over values of $m_{\Phi}$ between $10^{6} \mathrm{GeV}$ and $10^{7} \mathrm{GeV}$, and values of $\kappa$ from 0.5 to 5. The results are shown in figures 7 . The figures use $N_{\text {eff }}=3.62, f_{P Q}=10^{9} \mathrm{GeV}$ and a common scalar mass of $1 \mathrm{TeV}$. We also set $\alpha_{s}=0.11$ and use $g_{*}=61.75$. It is clear that it is easy to obtain dark matter abundances compatible with observations.

\section{Present Day Cosmic Axion Background Flux}

As we have discussed in the previous sections, dark radiation in the form of relativistic axions rarely interacts with its surrounding and the vast majority of the particles pass through the cosmos unperturbed, leaving a present day population of relic axions which is only diluted by the expansion of space. In this section, we compute the flux of axionic dark radiation in the current era (hence, 'today'), and find that it may even dominate over the flux of solar axions which are currently searched for by e.g. the CAST experiment at CERN. We furthermore point out that the differential axion spectrum and the conversion probability differs between solar axions and axions arising as dark radiation from moduli decay.

We re-emphasise here that while we focus for definiteness on axions, the same spectrum and flux will also hold for any other dark radiation candidate - for example hidden photons - that is produced by modulus decay.

Let us first discuss the flux of primordial axions arising from the instantaneous decay approximation for the modulus $\Phi$, thus constituting a mono-energetic population at all 

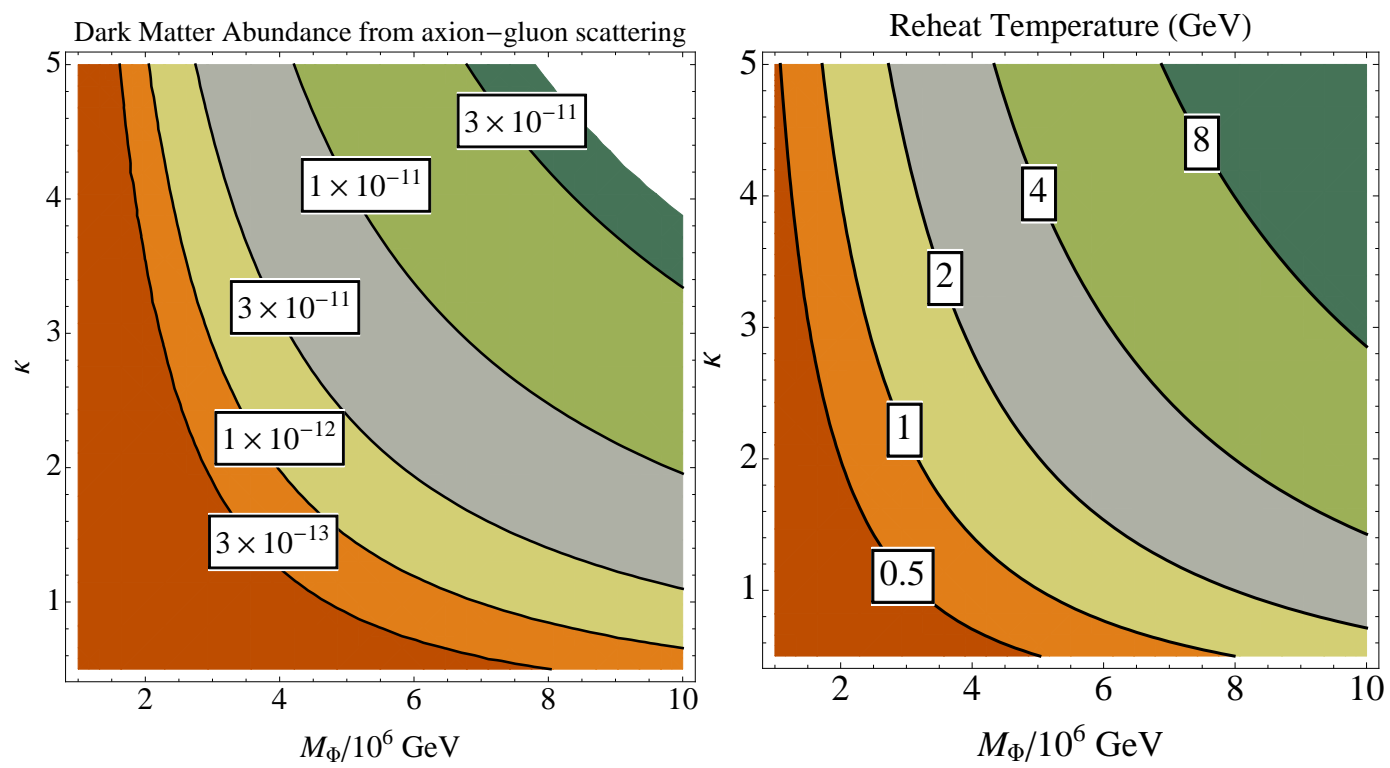

Figure 7: The dark matter abundance and reheating temperature as a function of $\kappa$ and $m_{\Phi}$. We have assumed $N_{\text {eff }}=3.62, f_{P Q}=10^{9} \mathrm{GeV}$ and a common squark and slepton mass scale of $1 \mathrm{TeV}$.

times. Immediately after the decay of $\Phi$, the population of axions is given by equation (2.7),

$$
n_{\text {axion }, 0}=\frac{B_{a} \kappa^{4}}{6 \pi^{2}} \frac{m_{\Phi}^{5}}{M_{P l}^{2}}=3.7 \cdot 10^{32} \cdot B_{a} \kappa^{4}\left(\frac{m_{\Phi}}{10^{6} \mathrm{GeV}}\right)^{5} \mathrm{~cm}^{-3}
$$

which we repeat here for clarity. Using the expression for $T_{r h}$ from equation (2.6), the entropy at time of reheating is easily found to be,

$$
s=\frac{2 \pi^{2}}{45} g_{*} T_{\text {reheat }}^{3}=\frac{2 \kappa^{3}}{45 \pi} g_{*}^{1 / 4}\left(\frac{5}{2}\left(1-B_{a}\right)\right)^{3 / 4} \frac{m_{\Phi}^{9 / 2}}{M_{P l}^{3 / 2}},
$$

where $g_{\star}=g_{*}\left(T_{\text {reheat }}\right)$. The axion abundance - both initially and at the present day is then

$$
\begin{aligned}
Y_{\text {axion }} & \equiv \frac{n_{a}}{s}=B_{a} \frac{45}{12 \pi} \frac{\kappa}{g_{*}^{1 / 4}}\left(\frac{2}{5\left(1-B_{a}\right)}\right)^{3 / 4} \frac{m_{\Phi}^{1 / 2}}{M_{P l}^{1 / 2}}= \\
& =3.85 \cdot 10^{-7} \frac{\kappa}{g_{\star}^{1 / 4}} \frac{B_{a}}{\left(1-B_{a}\right)^{3 / 4}}\left(\frac{m_{\Phi}}{10^{6} \mathrm{GeV}}\right)^{1 / 2}
\end{aligned}
$$

With the present day CMB photon density of $n_{\gamma}=413$ photons per $\mathrm{cm}^{-3}$, the axion number density today is given by,

$$
n_{a}=Y_{\text {axion }} s \simeq 7.04 n_{\gamma} Y_{\text {axion }}=1.12 \cdot 10^{-3} \frac{\kappa}{g_{\star}^{1 / 4}} \frac{B_{a}}{\left(1-B_{a}\right)^{3 / 4}}\left(\frac{m_{\Phi}}{10^{6} \mathrm{GeV}}\right)^{1 / 2} \mathrm{~cm}^{-3}
$$

The corresponding flux of dark radiation axions is then simply given by,

$$
\Phi_{a}=\frac{n_{a} c}{4}=8.39 \cdot 10^{6} \frac{\kappa}{g_{\star}^{1 / 4}} \frac{B_{a}}{\left(1-B_{a}\right)^{3 / 4}}\left(\frac{m_{\Phi}}{10^{6} \mathrm{GeV}}\right)^{1 / 2} \mathrm{~s}^{-1} \mathrm{~cm}^{-2} .
$$


For the example we have considered throughout this paper with $m_{\Phi}=5 \cdot 10^{6} \mathrm{GeV}, \kappa=1$, $g_{\star}=61.75$ and $B_{a}=0.145$, the present day flux is,

$$
\Phi_{a}=1.09 \cdot 10^{6} \mathrm{~s}^{-1} \mathrm{~cm}^{-2} .
$$

This flux may be compared to that of axions created from Primakoff scattering in the sun, which - contrary to the isotropic dark radiation flux of equation (5.6) - is always directional and suppressed by two powers of the axion decay constant [44],

$$
\begin{aligned}
\Phi_{\text {solar }} & =\left(g_{a \gamma \gamma} \times 10^{10} \mathrm{GeV}\right)^{2} 3.54 \times 10^{11} \mathrm{~cm}^{-2} \mathrm{~s}^{-1} \\
& \equiv 1.91 \times 10^{6}\left(\frac{10^{10} \mathrm{GeV}}{f_{a}}\right)^{2} \mathrm{~cm}^{-2} \mathrm{~s}^{-1} .
\end{aligned}
$$

The present day flux of dark radiation axions can easily dominate the solar axion flux for non-excluded values of the axion decay constant.

Moreover, the spectrum of solar axions differ significantly from that of axions produced from moduli decays. The differential solar flux spectrum is given by

$$
\frac{d \Phi}{d E}=\left(\frac{\alpha_{e m}}{\pi}\right)^{2} \frac{1}{f_{a}^{2}} 4.02 \times 10^{10} \mathrm{~cm}^{-2} \mathrm{~s}^{-1} \mathrm{keV}^{-1} \frac{(E / \mathrm{keV})^{3}}{e^{E /(1.08 \mathrm{keV})}-1},
$$

which peaks around $4 \mathrm{keV}$.

In the instantaneous decay approximation - which we will soon go beyond - the ratio of the axion energy to the CMB temperature is easily obtained. Initially, $E_{a}=$ $m_{\Phi} / 2$ and $T_{\gamma}=T_{\text {reheat }}$. The axion energy redshifts with the expansion of the universe as $E_{a} \sim \frac{1}{R}$, while the photon energies redshift as $T_{\gamma} \sim \frac{1}{g_{*}^{1 / 3} R}$. There are two boosts to the photon energy: from the reheat temperature to the time of neutrino decoupling, and from neutrino decoupling to now. These give

$$
\frac{T_{\gamma}}{E_{a}}=\left(\frac{g_{*}\left(T_{\text {reheat }}\right)}{g_{*}\left(T_{\nu \text { decoupling }}\right)}\right)^{1 / 3}\left(\frac{11}{4}\right)^{1 / 3}\left(\frac{T_{\gamma}}{E_{a}}\right)_{\text {reheat }},
$$

which evaluates to

$$
\frac{T_{\gamma}}{E_{a}}=\left(\frac{11}{4}\right)^{1 / 3} \frac{2 \kappa}{\pi}\left(\frac{1}{10.75}\right)^{1 / 3}\left(\frac{5\left(1-B_{a}\right)}{2}\right)^{1 / 4} g_{*}\left(T_{\text {reheat }}\right)^{1 / 12}\left(\frac{m_{\Phi}}{M_{P l}}\right)^{1 / 2} .
$$

Thus, in the instantaneous decay approximation, the energy of the dark radiation axions today is given by,

$$
E_{a}\left(T_{\gamma}\right)=1.97\left(\frac{M_{P l}}{m_{\Phi}}\right)^{1 / 2} \frac{T_{\gamma}}{\kappa g_{\star}^{1 / 12}\left(1-B_{a}\right)^{1 / 4}},
$$

where we have again abbreviated $g_{\star}=g_{\star}\left(T_{\text {reheat }}\right)$. In the example considered above with $m_{\Phi}=5 \cdot 10^{6} \mathrm{GeV}$, the present day energy of the axions is,

$$
E_{a}(\text { today })=238 \mathrm{eV}
$$


which is an order of magnitude lower than the energy of solar axions.

In the above discussion, we have used the instantaneous reheating approximation, where all moduli decayed at a time $\tau$. This produces a monoenergetic axion spectrum, with a single initial energy of $E_{a}=m_{\Phi} / 2$ that is subsequently redshifted. In reality the moduli decay gradually, and the axion energies are redshifted by different amounts depending on whether they come from early-decaying or late-decaying moduli.

We now want to determine the actual form of this spectrum. We note that this structure of the axion energy spectrum is essentially fixed by a time $t \sim$ (a few) $\tau$. At this point all the moduli will have decayed and the universe will have transitioned to radiation domination. As the axions are to a good approximation non-interacting, the energy spectrum now can be obtained simply by redshifting this spectrum.

To determine the structure of the energy spectrum we can calculate within a comoving volume: there is then no dilution in number density due to expansion of the universe. To convert to physical number densities, we simply multiply by a factor of $R^{-3}$ - which clearly does not affect the form of the energy spectrum.

For a modulus with lifetime $\tau$, the fractional decay rate is

$$
d N=-\frac{N d t}{\tau}, \text { giving } N=N_{0} e^{-\frac{t}{\tau}}
$$

The initial axion energy from a decay at time $t_{d}$ is always $E_{0}=\frac{M_{\Phi}}{2}$, and at time $t>t_{d}$ is

$$
E_{t}=E_{0}\left(\frac{a\left(t_{d}\right)}{a(t)}\right)
$$

Axions generated between times $t_{d}$ and $t_{d}+d t_{d}$ then lie in energies between $E_{t}$ and $E_{t}+d E_{t}$, where

$$
d E_{t}=\frac{\dot{a}\left(t_{d}\right)}{a\left(t_{d}\right)} E_{t} d t_{d} \equiv E_{t} H\left(t_{d}\right) d t_{d},
$$

and the number of axions generated between times $t_{d}$ and $t_{d}+d t_{d}$ is

$$
d N_{a}=\frac{2 B_{a}}{\tau} N_{0} e^{-\frac{t_{d}}{\tau}} d t_{d}=\frac{2 B_{a}}{\tau} N_{0} e^{-\frac{t_{d}}{\tau}} \frac{1}{E_{t} H\left(t_{d}\right)} d E_{t}
$$

At any time $t$, we then have the axion number spectrum

$$
\frac{d N}{d E_{t}}=\frac{2 B_{a}}{\tau} N_{0} e^{-\frac{t_{d}}{\tau}} \frac{1}{E_{t} H\left(t_{d}\right)}
$$

where $t_{d}$ and $H\left(t_{d}\right)$ are implicitly related to $E_{t}$ by $E_{t}=E_{0}\left(\frac{a\left(t_{d}\right)}{a(t)}\right)$.

The solution of eq. (5.15) requires the determination of $a(t)$. This can be found numerically as the modulus gradually decays and the universe transitions from matter domination to radiation domination. The relevant equations are

$$
\dot{\rho}_{\Phi}+3 H \rho_{\Phi}=-\frac{\rho_{\Phi}}{\tau}
$$




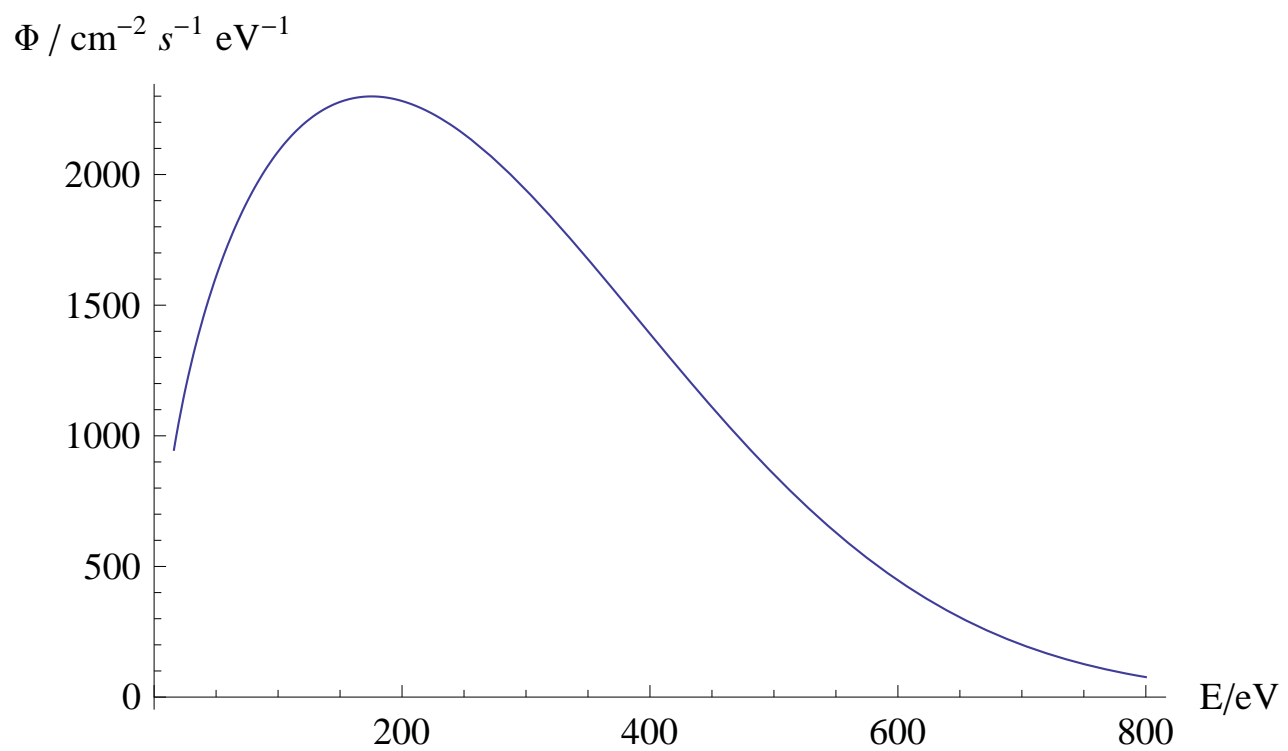

Figure 8: A typical axion flux per square centimetre per second, with $N_{\text {eff }}=3.62$. The integrated flux is $0.96 \times 10^{6} \mathrm{~cm}^{-2} \mathrm{~s}^{-1}$. The precise location of the energy peak depends on the value of $m_{\Phi}$. The cutoff at low energies is a numerical artefact from the spectrum computation.

$$
\begin{aligned}
\dot{\rho}_{\gamma}+4 H \rho_{\gamma} & =\frac{\rho_{\Phi}}{\tau} \\
H & =\sqrt{\frac{\rho_{\Phi}+\rho_{\gamma}}{3 M_{P l}^{2}}} .
\end{aligned}
$$

Using the numerical solution of $a(t)$, the resulting form of the axionic flux is shown in figure 8. The spectrum is normalised to $N_{\text {eff }}=3.62$. The exact location of the energy peak relative to the CMB temperature depends on $m_{\Phi}$, and a precise determination of this requires an analysis of Standard Model reheating beyond the instantaneous decay approximation. However for $m_{\Phi} \sim 10^{6} \div 10^{7} \mathrm{GeV}$ it will always be more energetic than the CMB peak by an approximate factor of $10^{6}$.

We can obtain analytic approximations using the simplifying assumption of either continual radiation domination or continual matter domination. These assumptions are accurate for either late modulus decays (radiation domination) or early modulus decays (matter domination).

For radiation domination, as $a \sim t^{1 / 2}$, we can relate $t_{d}$ and $E_{t}$ by

$$
\frac{t_{d}}{t}=\left(\frac{E_{t}}{E_{0}}\right)^{2} .
$$

giving a differential axion number density of

$$
\frac{d n\left(t, E_{t}\right)}{d E_{t}}=\frac{2 B_{a} N_{0}}{\tau E_{t}} e^{-\frac{t}{\tau}\left(\frac{E_{t}}{E_{0}}\right)^{2}} 2 t\left(\frac{E_{t}}{E_{0}}\right)^{2} .
$$


Continual matter domination with $a \sim t^{2 / 3}$ gives

$$
\frac{d n\left(t, E_{t}\right)}{d E_{t}}=\frac{2 B_{a} N_{0}}{\tau E_{t}} e^{-\frac{t}{\tau}\left(\frac{E_{t}}{E_{0}}\right)^{3 / 2}} \frac{3 t}{2}\left(\frac{E_{t}}{E_{0}}\right)^{3 / 2} .
$$

This spectrum is characteristic of dark radiation from moduli decays, and may be searched for by experiments like CAST that look for axion-like particles through conversion in a magnetic field. We note that since the Cosmic Axion Background flux arising from modulus decay is independent of $f_{a}$, the axion-to-photon generation rate in a magnetic

field scales as $f_{a}^{-2}$, as compared to the $f_{a}^{-4}$ fall-off of the axion-to-photon generation rate for solar axions.

\section{Conclusions}

In this article, we have studied the rich and interesting cosmophenomenology of axionic dark radiation. We have focused on three disparate areas: additional energy injection at $\mathrm{BBN}$ from axion-matter scattering, the late-time production of dark matter through scattering of axions into susy particles, and the relic Cosmic Axion Background flux today, which can be comparable to or larger than the solar axion flux. Our main point is that there is a rich phenomenology associated to axionic dark radiation, which is by no means sterile.

There are several clear directions for future work, one of which is the extension of our computation in section 4 to a larger number of processes for axion scattering into dark matter. In this initial study we principally considered the simplest case of universal s-channel gluon scattering. There are also many other diagrams, enumerated in figure 6. which we did not consider. These diagrams may in fact be more important, as the $a \gamma \gamma$ or agg vertex is generally suppressed by an extra factor of $\frac{\alpha}{4 \pi}$ compared to axionmatter vertices. Axion-matter-matter couplings do have a suppression by the mass of the relevant matter particle, but this should be less relevant once the centre of mass energy is comparable to the particle masses.

Furthermore, we have emphasised that bounds from overproduction of ${ }^{4} \mathrm{He}$ during BBN place interesting constraints on the axion decay constant and the reheating temperature. While our bounds in section 3.2 were derived for each decay channel separately and therefore constitute conservative bounds, a full, inclusive, analysis of the effects of dark radiation during $\mathrm{BBN}$ is likely to find even stronger constraints.

In section 5 we pointed out that dark radiation from modulus decays has a characteristic isotropic and non-thermal spectrum at an energy scale around six orders of magnitudes higher than that of the CMB. While we have focused on axions, the same spectrum would apply to other dark radiation candidates such as hidden photons, which may also interconvert to photons in the presence of a magnetic field. If it exists, this spectrum represents a Cosmic Axion Background which gives an image of the universe at a time $t \sim 10^{-6} \mathrm{~s}$, and its significance is obvious. As the flux is not dissimilar to the solar axion flux in magnitude, 
but isotropic in direction, it represents an exciting and worthwhile target for experiments such as CAST that rely on axion-photon conversion within a magnetic field.

\section{Acknowledgments}

JC is funded by a Royal Society University Research Fellowship and by the European Research Council starting grant 'Supersymmetry Breaking in String Theory'. DM was supported by the European Commission under the Marie Curie Initial Training Network UNILHC 237920 (Unification in the LHC era). Contents reflect only the authors' views and not the views of the European Commission. We thank Bobby Acharya, Stephen Angus, Erminia Calabrese, Jo Dunkley, Malcom Fairbairn and Andrew Powell for discussions related to this work.

\section{References}

[1] A. G. Riess, L. Macri, S. Casertano, H. Lampeitl, H. C. Ferguson, A. V. Filippenko, S. W. Jha and W. Li et al., "A 3\% Solution: Determination of the Hubble Constant with the Hubble Space Telescope and Wide Field Camera 3," Astrophys. J. 730 (2011) 119 [Erratum-ibid. 732 (2011) 129] [arXiv:1103.2976 [astro-ph.CO]].

[2] G. Hinshaw, D. Larson, E. Komatsu, D. N. Spergel, C. L. Bennett, J. Dunkley, M. R. Nolta and M. Halpern et al., "Nine-Year Wilkinson Microwave Anisotropy Probe (WMAP) Observations: Cosmological Parameter Results," arXiv:1212.5226 [astro-ph.CO].

[3] Z. Hou, C. L. Reichardt, K. T. Story, B. Follin, R. Keisler, K. A. Aird, B. A. Benson and L. E. Bleem et al., "Constraints on Cosmology from the Cosmic Microwave Background Power Spectrum of the 2500-square degree SPT-SZ Survey," arXiv:1212.6267 [astro-ph.CO].

[4] J. L. Sievers, R. A. Hlozek, M. R. Nolta, V. Acquaviva, G. E. Addison, P. A. R. Ade, P. Aguirre and M. Amiri et al., "The Atacama Cosmology Telescope: Cosmological parameters from three seasons of data," arXiv:1301.0824 [astro-ph.CO].

[5] P. A. R. Ade et al. [ Planck Collaboration], "Planck 2013 results. XVI. Cosmological parameters," arXiv:1303.5076 [astro-ph.CO].

[6] W. L. Freedman, B. F. Madore, V. Scowcroft, C. Burns, A. Monson, S. E. Persson, M. Seibert and J. Rigby, "Carnegie Hubble Program: A Mid-Infrared Calibration of the Hubble Constant," Astrophys. J. 758 (2012) 24 [arXiv:1208.3281 [astro-ph.CO]].

[7] S. H. Suyu, M. W. Auger, S. Hilbert, P. J. Marshall, M. Tewes, T. Treu, C. D. Fassnacht and L. V. E. Koopmans et al., "Two accurate time-delay distances from strong lensing: Implications for cosmology," Astrophys. J. 766 (2013) 70 [arXiv:1208.6010 [astro-ph.CO]].

[8] A. Ringwald, "Exploring the Role of Axions and Other WISPs in the Dark Universe," Phys. Dark Univ. 1 (2012) 116 [arXiv:1210.5081 [hep-ph]].

[9] K. Ichikawa, M. Kawasaki, K. Nakayama, M. Senami, F. Takahashi and , "Increasing effective number of neutrinos by decaying particles," JCAP 0705 (2007) 008 [hep-ph/0703034 [HEP-PH]]. 
[10] P. Graf and F. D. Steffen, "Thermal axion production in the primordial quark-gluon plasma," Phys. Rev. D 83 (2011) 075011 [arXiv:1008.4528 [hep-ph]].

[11] K. Nakayama, F. Takahashi and T. T. Yanagida, "A theory of extra radiation in the Universe," Phys. Lett. B 697 (2011) 275 [arXiv:1010.5693 [hep-ph]].

[12] W. Fischler and J. Meyers, "Dark Radiation Emerging After Big Bang Nucleosynthesis?," Phys. Rev. D 83, 063520 (2011) [arXiv:1011.3501 [astro-ph.CO]].

[13] J. Hasenkamp, "Dark radiation from the axino solution of the gravitino problem," Phys. Lett. B 707 (2012) 121 [arXiv:1107.4319 [hep-ph]].

[14] J. L. Menestrina and R. J. Scherrer, "Dark Radiation from Particle Decays during Big Bang Nucleosynthesis," Phys. Rev. D 85, 047301 (2012) [arXiv:1111.0605 [astro-ph.CO]].

[15] T. Kobayashi, F. Takahashi, T. Takahashi and M. Yamaguchi, "Dark Radiation from Modulated Reheating," JCAP 1203 (2012) 036 [arXiv:1111.1336 [astro-ph.CO]].

[16] K. S. Jeong and F. Takahashi, "Light Higgsino from Axion Dark Radiation," JHEP 1208 (2012) 017 [arXiv:1201.4816 [hep-ph]].

[17] M. Archidiacono, E. Giusarma, A. Melchiorri, O. Mena and, "Dark Radiation in extended cosmological scenarios," Phys. Rev. D 86 (2012) 043509 [arXiv:1206.0109 [astro-ph.CO]].

[18] C. Boehm, M. J. Dolan and C. McCabe, "Increasing Neff with particles in thermal equilibrium with neutrinos," JCAP 1212, 027 (2012) [arXiv:1207.0497 [astro-ph.CO]].

[19] T. Higaki, K. Kamada and F. Takahashi, "Higgs, Moduli Problem, Baryogenesis and Large Volume Compactifications," JHEP 1209 (2012) 043 [arXiv:1207.2771 [hep-ph]].

[20] K. Choi, K. -Y. Choi and C. S. Shin, "Dark radiation and small-scale structure problems with decaying particles," Phys. Rev. D 86, 083529 (2012) [arXiv:1208.2496 [hep-ph]].

[21] P. Graf and F. D. Steffen, "Axions and saxions from the primordial supersymmetric plasma and extra radiation signatures," arXiv:1208.2951 [hep-ph].

[22] M. Cicoli, J. P. Conlon and F. Quevedo, "Dark Radiation in LARGE Volume Models," arXiv:1208.3562 [hep-ph].

[23] T. Higaki and F. Takahashi, "Dark Radiation and Dark Matter in Large Volume Compactifications," JHEP 1211 (2012) 125 [arXiv:1208.3563 [hep-ph]].

[24] J. Hasenkamp and J. Kersten, "Dark radiation from particle decay: cosmological constraints and opportunities," arXiv:1212.4160 [hep-ph].

[25] K. J. Bae, H. Baer and A. Lessa, "Dark Radiation Constraints on Mixed Axion/Neutralino Dark Matter," arXiv:1301.7428 [hep-ph].

[26] K. S. Jeong and F. Takahashi, "Axionic Co-genesis of Baryon, Dark Matter and Dark Radiation," arXiv:1302.1486 [hep-ph].

[27] P. Graf and F. D. Steffen, "Dark radiation and dark matter in supersymmetric axion models with high reheating temperature," arXiv:1302.2143 [hep-ph].

[28] T. Higaki, K. S. Jeong and F. Takahashi, "A Parallel World in the Dark," arXiv:1302.2516 [hep-ph].

[29] M. Archidiacono, E. Giusarma, A. Melchiorri and O. Mena, "Neutrino and Dark Radiation properties in light of latest CMB observations," arXiv:1303.0143 [astro-ph.CO]. 
[30] C. Brust, D. E. Kaplan and M. T. Walters, "New Light Species and the CMB," arXiv:1303.5379 [hep-ph].

[31] C. Boehm, M. J. Dolan and C. McCabe, "A Lower Bound on the Mass of Cold Thermal Dark Matter from Planck," arXiv:1303.6270 [hep-ph].

[32] K. Choi, E. J. Chun and J. E. Kim, "Cosmological implications of radiatively generated axion scale," Phys. Lett. B 403, 209 (1997) [hep-ph/9608222].

[33] K. Choi, A. Falkowski, H. P. Nilles and M. Olechowski, "Soft supersymmetry breaking in KKLT flux compactification," Nucl. Phys. B 718 (2005) 113 [hep-th/0503216].

[34] B. S. Acharya, P. Kumar, K. Bobkov, G. Kane, J. Shao, and S. Watson, "Non-thermal Dark Matter and the Moduli Problem in String Frameworks," JHEP 0806 (2008) 064 [arXiv:0804.0863 [hep-ph]].

[35] R. Blumenhagen, J. P. Conlon, S. Krippendorf, S. Moster and F. Quevedo, "SUSY Breaking in Local String/F-Theory Models," JHEP 0909, 007 (2009) [arXiv:0906.3297 [hep-th]].

[36] J. R. Ellis, G. B. Gelmini, J. L. Lopez, D. V. Nanopoulos and S. Sarkar, "Astrophysical constraints on massive unstable neutral relic particles," Nucl. Phys. B 373, 399 (1992).

[37] S. Dimopoulos, R. Esmailzadeh, L. J. Hall and G. D. Starkman, "Limits On Late Decaying Particles From Nucleosynthesis," Nucl. Phys. B 311, 699 (1989).

[38] K. Jedamzik, "Big bang nucleosynthesis constraints on hadronically and electromagnetically decaying relic neutral particles," Phys. Rev. D 74 (2006) 103509 [hep-ph/0604251].

[39] M. Kawasaki, K. Kohri and T. Moroi, "Big-Bang nucleosynthesis and hadronic decay of long-lived massive particles," Phys. Rev. D 71 (2005) 083502 [astro-ph/0408426].

[40] G. Steigman, "Primordial Helium And the Cosmic Background Radiation," JCAP 1004, 029 (2010) [arXiv:1002.3604 [astro-ph.CO]].

[41] Y. I. Izotov and T. X. Thuan, "The primordial abundance of $4 \mathrm{He}$ : evidence for non-standard big bang nucleosynthesis," Astrophys. J. 710, L67 (2010) [arXiv:1001.4440 [astro-ph.CO]].

[42] E. Aver, K. A. Olive and E. D. Skillman, "A New Approach to Systematic Uncertainties and Self-Consistency in Helium Abundance Determinations," JCAP 1005 (2010) 003 [arXiv:1001.5218 [astro-ph.CO]].

[43] E. Aver, K. A. Olive and E. D. Skillman, "An MCMC determination of the primordial helium abundance," JCAP 1204, 004 (2012) [arXiv:1112.3713 [astro-ph.CO]].

[44] G. G. Raffelt, "Stars as laboratories for fundamental physics: The astrophysics of neutrinos, axions, and other weakly interacting particles," Chicago, USA: Univ. Pr. (1996) 664 p 